\section{THE GALACTIC SYSTEM*}

\section{By SIR JAMES JEANS, O.M., F.R.S.}

\begin{abstract}
THERE is a promontory on Mount Wilson from which the visitor to the Observatory can look down at night and see the lights of Pasadena and Los Angeles on the plain 7,000 ft. below. He cannot distinguish individual lights, but sees two patches of luminosity which indicate the outlines of the cities and the distribution of density-if not of population, at least of the street-lights. It is so easy to study this distribution from up here, and so difficult from inside the cities, where one cannot see the forest for the trees.
\end{abstract}

This is, of course, an astronomical parable. In the night sky we see a number of distinct stars, and also a far greater number which are merged into the continuous band of light we call the Milky Way. These together constitute the galactic system-the city of stars in which we reside. But far out beyond this we see objects of another kind-the extra-galactic nebulæ - which we believe to be other cities of stars, external to our own.

It is easy to study the distribution of light, and so of stars, in these external galaxies; we need only take a photograph or, better, photometric measurements, and the thing is done. But our own galaxy presents problems of an entirely higher order of difficulty.

If the stars were distributed uniformly through infinite space, the number in a sphere of any size would, of course, be proportional to the cube of the radius of the sphere, and this would lead to the simple law that vision down to stars of one magnitude fainter would increase the number of stars which could be seen fourfold. If the uniform distribution of the stars fell off after a certain distance, this distance would be revealed by the failure of this law. Using this method, the two Herschels studied the distribution of the stars in space, and were led to picture the galactic system of stars as a flat disk, the plane of the Milky Way being, of course, the plane of the disk. This plane is observed to divide our sky into exactly equal halves, and the sky looks about equally bright in all directions in it; whence the Herschels concluded that we are close to the centre of the disk.

At a later date, the globular clusters seemed to tell a different story. These are compact clusters of millions of stars, all being very similar in appearance. So far back as 1911, A. R. Hinks had noticed that they all lie in one half of the sky. A detailed study by Shapley confirmed this-nearly all lie within a range of $130^{\circ}$ of galactic longitude. Cepheid variables abound in the clusters, so that their distances are easily measured, and Shapley could map out the distribution of the clusters in space. He found that all 'except one-possibly a stray-lie in or near a circle of about 100,000 light-years radius, in the galactic plane. But the sun is not at or near the centre of this circle; it is about 40,000 light-years distant. It was natural to think that the boundary of this system of clusters must mark out the limits of the galaxy, but it seemed strange at the time that this did not coincide with the boundary of the visible stars.

The discovery that space is filled with obscuring matter has now removed this difficulty, and brought all

- Abstract of a Royal Institution discourse delivered on April 28. the elements of the problem into harmony. Observation shows that this obscuring matter makes a fog of which the density varies greatly in the different parts of space. As might be expected, it is densest in the galactic plane; here a beam of light is halved in intensity after traversing about 3,000 light-years of distance. Thus the range of visibility in the fog is not a very great number of thousands of light-years. The Herschels, and many after them, who did not know of the fog, mistook the range of visibility in the fog for the radius of the galaxy. This is why we seemed to live at the centre of things; for in a fog, we each have our own sphere of vision, of which we are always at the centre.

The figure just mentioned shows that the fog reduces light to a ten-thousandth part of its original intensity in travelling from the centre of the galactic system to the sun. Thus individual stars near this centre are invisible to us, and we see less than a half of the whole system of stars. For the same reason, we cannot see external objects which lie in or near the galactic plane; there is too much obscuring matter between them and us for their light to get through.

If the fog were much denser than it actually is, we should see only the stars in our immediate proximity, and a few of exceptional brightness beyond. If we were unaware of the fog, we should conclude that there is an excessive concentration of stars in our immediate proximity. Astronomers made exactly this mistake for a time, thinking that we lived in the midst of a 'local eluster' of exceptionally bright stars. Now that we know of the fog, we can allow for its effects and, for any assumed density of fog, can calculate the arrangement of stars which will exactly fit the observations. If we underestimate the fog, we shall obtain a 'local cluster'; while if we over-estimate the fog, we shall get the opposite result, namely, that stars are exceptionally few in our neighbourhood, so that we are living in a 'hole'. Actually both results have been obtained in recent years by different investigators, but the simplest interpretation of their results is, I think, that they have respectively underestimated and over-estimated the density of fog. There are rather strong reasons for thinking that there can be neither a 'local cluster' nor a 'local hole' -these are, in brief, that the whole galactic system is rotating with different speeds in different parts, so that both clusters and holes would soon be smoothed out. Thus the most likely value for the coefficient of absorption by the fog would seem to be that which just gets rid of the 'local cluster' without replacing it by a 'local hole', and this is about equal to the value mentioned above.

For a long time it was something of a puzzle to understand why a disk-shaped group of stars such as our galaxy should not all fall together at its centre of gravity, and it has often been suggested that our galaxy must be in rotation, as many of the external galaxies are known to be. In 1913 Poincaré calculated that our galaxy could be saved from this fate if it rotated about once every 500 million years. In the same year, Charlier found that the invariable plane of the solar system appeared to be moving against the background of the stars. Now the solar system keeps its invariable plane always fixed in the same direction, just as a spinning gyrostat does, so that, as Eddington immediately pointed out, the apparent motion found by Charlier could only mean that the background of stars was not at rest but was itself in 
motion. Such a movement of the background is now one of the well-established facts of astronomy.

A superficial study of the nearer stars suggests that they are moving at random, with differing speeds and in different directions. But a careful statistical investigation reveals law and order in the motions, not of individual stars but of statistical groups. The motion is best described by the statement that each group of stars (sufficient in number to justify statistical treatment, but also comprised within a sufficiently small volume of space) is describing an orbit about a centre. This centre is the same for all groups, and coincides exactly with the centre of the galaxy, as determined by Shapley from the arrangement of the globular clusters. Those groups which are farthest from the centre move most slowly, just as, in the solar system, those planets which are farthest from the sun move most slowly. The reason is, of course, that each star describes an orbit under the gravitational force of the rest of the stars, just as each planet describes an orbit under the gravitational force of the rest of the solar system.

Detailed statistical study of the stars near the sun shows that, on the average, the orbital speed of a star falls off by $1 \mathrm{~km}$. a second for every 200 lightyears increase of distance from the centre of the galaxy. This single datum, which is quite well determined, shows that the sun must take about 250 million years to perform its journey round the centre of the galaxy. Thus it must have completed some ten or a dozen orbits since the earth was born. If the sun is at a distance of 40,000 light-years from the centre of the galaxy, then it must describe its orbit at a speed of about $300 \mathrm{~km}$. a second, a conclusion which agrees well enough with independent estimates made by spectroscopic measurements of the speed of the sun relative to the external galaxies and remote globular clusters.

The various data which have just been mentioned provide the means for weighing the mass which keeps the sun in its orbit, and so the galaxy as a whole. Estimates vary from 110,000 million to 180,000 million times the mass of the sun, so that it seems safe to say that the galaxy contains hundreds of thousands of millions of stars, although the majority are rendered invisible by the thick layer of fog which lies between them and us. It used to be thought that our galaxy was more massive than the others we see in the sky, but this no longer appears to be the case. We are familiar with groups of stars which are held together by their mutual gravitational attractionsthe globular clusters provide an instance. There are also clusters of nebulæ which are held together in the same way. It is possible to determine the speeds of motion of the individual nebulæ of a cluster, and so deduce the gravitational forces needed to hold the cluster together. In this way, the average galaxy is found to have a mass of the order of from 100,000 million to 200,000 million suns. Thus there can be no doubt that the external galaxies are at least comparable with our own galaxy in mass.

Finally, it used to be thought that the external galaxies are substantially smaller than our own in size, but it has recently emerged that this too is fallacious. We only see a small part of a galaxy when we study its apparent size on a photographic plate; there is a much larger part beyond, which can only be detected by delicate photometric measurements. When we take this into account, the galaxies still show considerable differences in size, but the majority of the larger prove to be comparable with our own. On the whole, then, our galaxy is simply one of many similar galaxies. Probably about four million such can be seen photographically in the great Mt. Wilson telescope. If we allot 100,000 million stars to each, this makes a total of about $4 \times 10^{17}$ stars-a large number, although still small in comparison with the number of molecules in a cubic centimetre of ordinary air.

\section{REGIONAL PLANT ECOLOGY IN THE UNITED STATES}

\author{
By Dr. V. J. CHAPMAN \\ Botany School, Cambridge
}

$\mathrm{T}$

HE last four years has seen the publication, by J. H. Davis, of three important papers on the vegetation of Southern Florida*. These three valuable papers yield a clear picture of the vegetation and its interrelations with soil types, elimate and physio. graphy. The area is especially interesting because climax vegetation is normally related to the climate, but Davis establishes a good case in this area for relating it primarily to the physiography. If the author continues these studies - as one sincerely hopes he will-the vegetation of Southern Florida will be known and understood in very considerable detail. This will be no mean feat for an area of such size. The majority of ecological studies usually refer to relatively restricted areas, and it is refreshing to find a study that embraces such a large region. A broad survey with accompanying detailed studies opens up major problems that would not be so evident in a study of a small area.

In the first paper, on the mangrove vegetation, Davis points out that they are primarily edaphic forests, a conclusion with which I agree, though I would add that there is also a physiographic element involved. Tropical forest of the 'ham. mock' type is regarded as the climax vegetation, though in the third paper it is also, albeit incorrectly, implied that mangroves represent a climax type. There is no evidence of a transition to brackish or freshwater marsh such as may be found in Jamaica. Davis studied the environmental factors in some detail and he shows that the mangrove species possess a wide tolerance of salinity and grow on at least four types of soil, one of which is a marine peat. I have arrived at similar conclusions from work in Jamaica. Davis relates the mangrove zonation to the height of the surface water, but here one feels that more evidence would be desirable. Some exceedingly interesting information is provided about dispersal. 10,000 Rhizophora seedlings are estimated to float towards the Tortugas every year; this represents only a proportion of the total crop because about 50 per cent of the seedlings from a tree remain embedded in the mud beneath it. The rate of sur. vival at the end of one year is 50 per cent for Rhizophora, 30 per cent for Avicennia and 20 per cent for Laguncularia.

One of the important features of these three papers is the great use made of aerial photography in the study of the communities and the preparation of the

* Davis, J. H., "The Ecology and Geologic Rôles of Mangroves in Florida", Carn. Inst. Wash. Pub., 517 (1940); "The Ecology of the Vegetation and Topography of the Sand Keys of Florida", Carn Inst. Wash. Pub. $524(1942)$; The Natural Features of Southern
Florida", State of Florida Dept. of Conservation, Bull. 25 (1943). 